\title{
Inmigración y salud mental infanto-juvenil: análisis de los artículos publicados en revistas nacionales de Psicología durante el periodo 2003-2013
}

\section{Immigration and infant-juvenile mental health: an analysis of articles published in national Psychology journals during the 2003-2013}

\author{
Gabriel Abarca Brown ${ }^{1}$ \\ Escuela de Psicología, Universidad de Santiago, Santiago, Chile \\ Laboratorio Transdisciplinar en Prácticas Sociales y Subjetividad (LaPSoS), Universidad de Chile, Santiago, \\ Chile \\ Mauricio Carreño Hernández \\ Escuela de Psicología, Universidad de Santiago, Santiago, Chile \\ Programa Adultos COSAM Colina, Santiago, Chile
}

(Rec: octubre 2014 - Acep: noviembre 2014)

\begin{abstract}
Resumen
El presente artículo tiene por objetivo describir el estado actual de las publicaciones en revistas de Psicología a nivel nacional respecto del tema migratorio, particularmente de la salud mental en población infanto-juvenil inmigrante. Para ello se revisaron 1.094 artículos publicados en seis revistas de Psicología en Chile durante el periodo 2003-2013. Se encontraron 26 artículos referidos a temas migratorios, los que posteriormente fueron clasificados según: tipo de investigación, proyecto asociado, apoyo institucional, nivel de análisis, población objetivo, y contexto del estudio. Los resultados indican una baja tasa de publicación en temas migratorios, y la inexistencia de artículos sobre inmigración y salud mental infanto-juvenil. Finalmente, se enfatiza en la necesidad de publicaciones en Psicología que aborden la temática, contemplando dimensiones socioculturales en sus análisis.
\end{abstract}

Palabras clave: publicaciones en Psicología, migración, salud mental en población infanto-juvenil inmigrante.

\begin{abstract}
This paper aims to describe the current state of the national publications in journals of Psychology respect to the subject of immigration, particularly mental health in infant-juvenile immigrant population. In order to do so, 1.094 articles published in six journals of Psychology in Chile during the period 2003-2013 were reviewed. Twenty-six articles on immigration were found, which were subsequently classified by: type of research, associated project, institutional support, level of analysis, targeted population, and context of study. The results indicate a low rate of publication on the subject of immigration issues, and the lack of articles on immigration and infant-juvenile mental health. Finally, it is emphasized the need for publications in Psychology to address immigration, considering socio-cultural dimensions in their analysis.
\end{abstract}

Keywords: publications in Psychology, migration, mental health in infant-juvenile population.

\footnotetext{
${ }^{1}$ Correspondencia dirigida a : Gabriel Abarca Brown. Escuela de Psicología, Universidad de Santiago de Chile. Av. Ecuador 3650, 3er piso, Estación Central, Santiago. E-mail: gabriel.abarca@usach.cl.
} 


\section{Introducción}

En las últimas dos décadas el número de inmigrantes que viven en Chile se ha triplicado. Según el censo realizado el año 2012, los extranjeros que residen en el país son 339.536 , lo que representa un $2 \%$ de la población general (INE, 2013). Más allá de la tasa de extranjeros -distante considerablemente a la de algunos países europeos o asiáticos- resaltan ciertos cambios que a nivel sociodemográfico se han producido en la conformación de estos grupos migrantes. Si bien en la primera mitad del siglo XX los/as inmigrantes eran de origen predominantemente europeo, en la actualidad se observa que más del $70 \%$ proviene de países sudamericanos. En el escenario actual, destacan ciudadanos de nacionalidad peruana (30,52\%), argentina $(16,79 \%)$ y colombiana (8.07\%), principalmente (INE, 2013). A esto se suma, que quienes han arribado en los últimos años ya no tienen como horizonte las oportunidades de negocio e inversión -como los inmigrantes europeos de principios de siglo XX- sino que buscan mejorar sus condiciones de vida e ingreso económico a través de la incorporación a labores relacionadas con la mano de obra en áreas como la construcción, la industria y los servicios domésticos (Stefoni \& Fernández, 2012; Tijoux, 2013).

Sin embargo, las expectativas de los/as actuales inmigrantes no siempre se cumplirían. Muy por el contrario, parte de los/as extranjeros encuentran una serie de limitaciones -jurídicas, sociales, habitacionales, sanitarias, entre otras- que los/as dejan en una condición de vulnerabilidad psicosocial que se traduce la mayor parte de las veces en un deterioro de sus vidas cotidianas y, particularmente, de su salud mental (Vázquez-De Kartzow, 2010).

Si bien en Chile no existen datos concluyentes en la materia, un estudio realizado el año 2011 sobre prevalencia de trastornos mentales en población inmigrante adulta e infanto-juvenil (Rojas, Fritsch, Castro, Guajardo, Torres, \& Díaz, 2011), reveló algunas cifras que sitúan a esta última en un lugar preocupante: por un lado, la prevalencia de trastornos en la población infanto-juvenil (hijos de inmigrantes nacidos en Chile) es más alta (29,3\%) que en la población adulta consultante (17,8\%); mientras que por otro, dicha población encuentra en el sistema de salud chileno una serie de barreras relacionadas con la desinformación sobre derechos, así como también limitaciones de orden económico, sociocultural y/o idiomático (Alvarado, 2008).

Los datos entregados por esta investigación confirman literatura internacional que indican que la segunda generación de inmigrantes tiende a presentar mayores problemas de salud mental que la de sus padres (Harker, 2001; Zolkowska, Cantor-Graae, McNeil, 2001); y que las tasas de prevalencia de trastornos mentales tienden a igualarse a la de los países de acogida (Breslau, AguilarGaxiola, Borges, Kendler, Su, Kessleret, 2007).

Diversas disciplinas pertenecientes al campo de las ciencias sociales han intentado comprender los problemas asociados a los movimientos migratorios. La Sociología, la Antropología, la Economía Política y la Educación, principalmente, parecen destinar -en términos generales- mayores esfuerzos con el objeto de analizar los problemas asociados a la inmigración y la población infanto-juvenil (por ej. "vida cotidiana", "racismo", "discriminación", entre otros). Del mismo modo, estudios emanados desde las Ciencias de la Salud han logrado establecer las coordenadas generales para el trabajo en salud mental. Así, la Psiquiatría y la Salud Pública han contribuido en la identificación y prevalencia de cuadros sintomáticos asociados a procesos migratorios (Cano \& Soffia, 2009). No obstante, a simple vista, pareciera no existir el mismo interés por estos temas en el campo de la Psicología, y menos por asuntos asociados a la salud mental de la población infanto-juvenil inmigrante.

En función de los antecedentes anteriormente expuestos, surge la pregunta: ¿cuál es el estado del arte en investigación en Psicología sobre temas migratorios y población infanto-juvenil? En tal sentido, el objetivo del presente artículo es describir el estado actual de las publicaciones en revistas de Psicología a nivel nacional respecto del tema migratorio, particularmente de la salud mental en población infanto-juvenil inmigrante. Para alcanzar este objetivo, se realiza un análisisdescriptivo de los artículos publicados en seis revistas de Psicología a nivel nacional que destacan por su nivel de indexación y/o tradición.

Conocer las publicaciones en el campo de la Psicología relativas al fenómeno migratorio es particularmente relevante debido a que, comúnmente, los asuntos migratorios son abordados en sus aspectos macrosociales, vale decir, en sus dimensiones demográficas y/o político-económicas. En cambio, los aspectos microsociales asociados, por ejemplo, vida cotidiana y/o salud mental en población infanto-juvenil, tienden a ser invisibilizados o escasamente priorizados en los análisis (Jensen, 2008).

Asimismo, se torna relevante conocer el estado actual de la investigación en el campo de la Psicología, y particularmente de la salud mental, si se considera que la vida cotidiana -tanto de algunas familias como 
de algunos/as niños/as y jóvenes inmigrantes- está muchas veces enmarcada dentro de condiciones sociales que promueven la vulneración de derechos, afectando consecuentemente su calidad de vida.

\section{Antecedentes contextuales de la inmigración infanto-juvenil: cadenas de cuidado, hogares transnacionales y reconfiguración de familiar}

Los estudios sobre migración han revelado, tanto a nivel nacional como internacional, que una de las principales características de los movimientos fronterizos contemporáneos es la llamada "feminización" de los flujos migratorios. De hecho, a juicio de Castles y Miller (2004), la "feminización de las migraciones" se constituiría en uno de los cinco rasgos de la llamada "Era de la Migración". Por este fenómeno -cuya visibilización comenzó durante la década de los ochenta- se entiende el creciente número de mujeres autónomas que salen de sus países con intereses distintos a la reagrupación familiar, dejando familias dependientes en sus países de origen (Acosta, 2012).

La salida de las mujeres en busca de un mejoramiento en las condiciones de vidas tanto individuales como familiares, ha tensado una de las prácticas históricamente atribuidas al mundo femenino, a saber: el cuidado de los otros. Los procesos migratorios actuales han generado fracturas en las formas tradicionales de cuidado, al introducir lógicas temporo-espaciales que no se reducen a la relación inmediata entre los sujetos. La también denominada "crisis del cuidado" ha conducido al surgimiento de "cadenas globales de cuidado", es decir, una serie de vínculos personales -principalmente familiares- basadas en una labor de asistencia (Arriagada \& Moreno, 2012). Estas cadenas se conforman con el objetivo de sostener la vida cotidiana tanto de las familias que quedan en los países de origen como de quienes, en el plano laboral, son objeto de los cuidados de los inmigrantes en los países de destino (Pérez, 2010).

Una de las principales características de estas cadenas de cuidado de carácter transnacional son las profundas desigualdades que se evidencian en sus polos. Por un lado, es posible constatar a un grupo -por lo general perteneciente a un país o región con mayor desarrollo económico- con capacidad de delegar a otros las labores de cuidado; mientras que por otro, un grupo que cuida y que, paradojalmente, puede sufrir la pérdida de parte de su propio cuidado. Así, la transferencia de cuidado desde sectores con mayor poder por sobre otros, revela una dimensión política en estas prácticas, que reproduce diferencias en el plano de lo social al jerarquizar a las mujeres y familias según clase, género, raza, entre otras.

Entre las condiciones que posibilitan el aumento de demanda de cuidado se encuentra: a) el envejecimiento de la población y aumento de la esperanza de vida de las personas; b) los cambios en la estructura y en las formas de vida que limitan la disponibilidad de cuidadores; y c) la evolución de los sistemas formales de cuidado, como por ejemplo, alta precoz hospitalaria, reformas de atención psiquiátrica, entre otras (Arriagada \& Moreno, 2012). Asimismo, vale la pena señalar que esta reorganización social de los cuidados estaría condicionada por la debilidad de los sistemas de protección social, el papel de los hombres en las prácticas de cuidado, la precariedad del mundo laboral donde se insertan la población migrante, y la política migratoria en términos globales.

De esta forma, la "feminización de las migraciones" ha dado lugar en los últimos treinta años a reconfiguraciones familiares que en la actualidad son denominadas "hogares transnacionales". Esto se refiere a hogares en donde la mujer autóctona es sustituida por la mujer inmigrante, y esta última por otras mujeres -por lo general del núcleo familiar- quienes quedan a cargo de sus hijos y/o dependientes en el país de origen.

La feminización de las migraciones ha traído consigo una serie de transformaciones no sólo a nivel transnacional, sino también al interior de los hogares en los países de origen y de destino, cuestión que, por lo demás, no se tiende a observar necesariamente cuando es el hombre quien migra. En el caso de las mujeres, estos reordenamientos provocan, por un lado, una redistribución del trabajo de cuidado entre los familiares que quedan a cargo en el país de origen, y por otra, una gestión del bienestar que asume nuevas coordenadas (Pérez, 2009). La realidad social de las familias inmigrantes no se reduce entonces a un sólo territorio, sino que asume acciones que encuentran cierta legitimación en una institución donde los miembros de dicha familia son capaces de mantener vínculos de pertenencia a pesar de las distancias, de los riesgos y desestabilizaciones que el sistema familiar pueda sufrir (Acosta, 2012).

Las transformaciones a nivel de las cadenas de cuidado y las reconfiguraciones evidenciadas en los hogares de personas migrantes no son en absoluto ajenas a la realidad chilena. Los estudios disponibles en el campo de las ciencias sociales así lo han demostrado, 
particularmente, en sus investigaciones con población peruana inmigrante (Cano \& Soffia, 2009).

El flujo inmigratorio en las últimas décadas a nivel local se ha caracterizado por el predominio del número de mujeres que cruzan la frontera. Algunas de ellas, encuentran en trabajos de carácter doméstico un espacio laboral que les permite sostener tanto sus cotidianidades como la de sus familias en los países de origen. Estas premisas son respaldadas por cifras como la de la encuesta CASEN (MIDEPLAN, 2011), que revela que durante la década de los noventa la participación en el servicio doméstico de mujeres chilenas bajó de un $7,6 \%$ a un $4,7 \%$. Esta retirada ha dado lugar a que las mujeres inmigrantes ocupen gradualmente dichos empleos. De hecho, desde el año 2006 al 2011, los/ as inmigrantes que desarrollan labores en el ámbito doméstico han crecido de un 3,7\% a un 5,6\%.

El incremento de la población inmigrante en empleos domésticos y la consecuente formación de cadenas globales de cuidado, ha generado un desbalance entre las prácticas de cuidado que se llevan a cabo en los países de destino y los países de origen. En términos promedio, la evidencia muestra que los/as niños/as migrantes tienden a permanecer en sus países de origen durante cinco años separados de sus madres y/o padres, al cuidado de otras personas (abuelas, tías, u otros).Si bien existe por parte de sus padres la voluntad de reencontrarse con sus hijos/as, muchas veces se ven impedidos por las condiciones de vida, particularmente de habitabilidad (trabajo en formato puertas adentro, hacinamiento, entre otros) (Cortez, 2004; Vásquez, 2004). Lo anterior es respaldado por el Servicio Jesuita a Migrantes (2010, en Pavez, 2013) que señala que del total de personas atendidas en dicho lugar, un $66,6 \%$ tiene por lo menos un hijo/a en su país de origen. Así, las familias migrantes tienen en promedio a 2,3 hijos/ as en el país de origen frente a 1,7 en Chile.

Fue recién a partir del año 2004 cuando se comienza a registrar procesos de reunificación de familias inmigrantes en Chile (MIDEPLAN, 2006). Habitualmente, dichos procesos se realizan de modo informal (luego de los viajes de fin de año o vacaciones) porque no existe una legislación específica al respecto dentro de las políticas migratorias actuales (Cortez, 2004; Stefoni, Acosta, Gaymer, Casas-Cordero, 2008; Vásquez, 2007).

Según los datos proporcionados por la medición censal del año $2002^{3}$, el grupo de inmigrantes menores

\footnotetext{
3 Algunos datos del censo del año 2012 aún no se encuentran disponibles en el Servicio Nacional de Estadísticas (INE). Por tanto, se optó por la utilización de los datos de la medición censal anterior (año 2002).
}

de catorce años es el tercer grupo con mayor presencia a nivel nacional con 34.006 personas (INE, 2003). Esto evidencia la alta presencia que la población infantojuvenil inmigrante tiene en Chile, donde predominan los/as niños/as de nacionalidad peruana (Ministerio del Interior y Seguridad Pública, 2009).

\section{Marco Teórico}

\section{Vida cotidiana y aspectos subjetivos de niños/as inmigrantes}

Si bien en los últimos años los estudios han evidenciado una diversificación de los países de origen de los inmigrantes, quienes se han constituido como el principal grupo de extranjeros en Chile son los ciudadanos de nacionalidad peruana. Por lo mismo, gran parte de las investigaciones realizadas en materia de migración, y con particularidad los referidos a infancia e inmigración, han recaído en esta población.

La migración peruana, tal como revelan los estudios realizados por Stefoni $(2003,2012)$, se ha caracterizado por la formación de grupos transnacionales basados en relaciones de parentesco. En tal sentido, las primeras generaciones de inmigrantes dejan tras de sí una comunidad de origen con la que mantienen vínculos basados, fundamentalmente, en el envío de dinero para el sostén económico de las familias. En los casos en que la reunificación familiar puede ser llevada a cabo, el ámbito escolar se convierte para los niños/as inmigrantes en el terreno de confrontación por excelencia, espacio donde las relaciones de poder son desplegadas y donde el/la infante encuentra las puertas de entrada para su ingreso a la cultura chilena.

$\mathrm{Al}$ respecto, las investigaciones desarrolladas por Tijoux $(2013 ; 2013 b ; 2012)$ han contribuido en la comprensión de las cotidianidades del mundo infantil inmigrante en las escuelas chilenas. En su trabajo "Las escuelas de la inmigración en la ciudad de Santiago. Elementos para una educación contra el racismo", Tijoux (2013) sostiene que los hijos de padres peruanos presentan una condición heredada, vale decir, sufren las consecuencias de la migración de sus padres enmarcada en contextos migratorios de pobreza y precariedad. Los niños/as portan una culpa que proviene de sus orígenes.

De esta forma, los "hijos de estos inmigrantes, sin buscarlo, quedan vestidos de una condición especial y espacial: ser hijos de la inmigración como problema arraigado en la pobreza de su clase social y portar 
la extranjeridad de un país que provoca resquemor y crítica. A partir de esta doble negación, todas las acciones familiares $o$ individuales serán evaluadas negativamente" (Tijoux, 2013, p. 294).

Siguiendo los planteamientos de Tijoux (2013), los niños/as peruanos vivencian en las escuelas las burlas de los/as niños/as y profesores/as chilenos/as por sus costumbres. Esto se evidencia con particular fuerza en las recriminaciones y correcciones que sufren por su modo de hablar, la que, gran parte de las veces, es concebida como indicador de deficiencia intelectual.

Asimismo, Hevia (2009), en sus estudios de niños/ as peruanos, ha destacado que estos son concebidos como "problemáticos" y "respondones" tanto por sus compañeros/as como por sus profesores/as. En razón de esto, se ven obligados a desplegar una serie de mecanismos y estrategias de adaptación con el objeto de lograr un mejor grado de ajuste con la cultura chilena. Tal es el caso de la utilización del acento peruano sólo en el contexto doméstico, tomando distancia de los modismos chilenos al hablar con sus familiares o compañeros peruanos. Sus familias buscan transmitir cierta capacidad que les permita discriminar entre los elementos que pueden adoptar de sus pares chilenos, $\mathrm{y}$ aquellos que desean que mantengan como parte de sus tradiciones.

Por otro lado, la misma autora, ha identificado la existencia de un trato diferencial en función del color de la piel (Hevia, 2009). Es decir, una valoración distinta que se da entre los estudiantes por los rasgos que remiten a "lo indígena". Esta constatación es también relevada por Tijoux (2013b), quien sostiene que tanto las familias como los/as niños/as inmigrantes específicamente, son muchas veces discriminados por su color, tildados de "cholos", "indios" o "negros", lo que afecta la representación que tienen de sí y de la comunidad chilena. Esto último, es coherente con la evidencia rescatada por la UNICEF (2004), que señala que los/as niños/as inmigrantes tienden a percibir negativamente sus rasgos y a expresar una valoración positiva de los rasgos corporales de los chilenos.

Otros estudios, en cambio, han apuntado a la condición habitacional y sus efectos en la vida cotidiana. Algunos/as inmigrantes vivencian el hacinamiento -en especial durante los primeros años- debido a las condiciones de precariedad y marginalidad al momento del ingreso al país. Tal como han mostrado los estudios de Pavez en población peruana (2013; 2010), esto se vuelve particularmente sensible en los niños/as, quienes son objeto de las consecuencias de los conflictos provocados entre las familias que comparten espacios reducidos, atentando y vulnerando sus derechos de privacidad y dignidad.

Por último, en su estudio, Pavez (2010) también contempla la relación que mantienen las familias inmigrantes con las instituciones gubernamentales (servicios, oficinas de gobierno, etc.). La autora plantea que los/as funcionarios/as de dichas instituciones muchas veces desconocen los derechos de los/as niños/ as inmigrantes, particularmente los referidos a la no discriminación.

De esta forma, los espacios donde es posible identificar la discriminación y la vulneración de derechos con mayor frecuencia son: el barrio, la escuela, el grupo de pares, los centros de salud; instancias en las que diversos actores reproducen la marginalidad y violencia de la que son objeto las familias, y particularmente, los niños/as inmigrantes (Cortez, 2007; Pavez, 2010; Reyes, 2007). En reiteradas ocasiones, los niños/as son objeto de maltratos físicos y psicológicos que, por un lado, desfavorecen su integración a las nuevas comunidades, y por otro, afectan su estabilidad emocional, dando paso a la aparición de sentimientos de abandono y angustia (Stefoni et al., 2008).

\section{Salud mental en población infanto-juvenil inmigrante y servicios de salud}

Los escasos datos existentes a la fecha en Chile han puesto en evidencia la agudización de las problemáticas de salud mental en población inmigrante infanto-juvenil. La tasa de trastornos mentales en niños/as alcanzaría el 29,3\%, vale decir, el doble de la población adulta. Entre dichos trastornos prevalecerían los cuadros anímicos (14,3\%) y de ansiedad (5,7\%). Adicionalmente, el 36,1\% de los/as apoderados/as de estos niños/as y adolescentes perciben que quien está a su cargo presenta algún problema de salud mental (Rojas et al., 2010).

Sin embargo, la respuesta a nivel institucional, especialmente en la formulación y puesta en marcha de programas de salud pertinentes a la realidad vivenciada por las familias inmigrantes, no ha sido la más óptima. Según el informe "Diagnóstico y factibilidad global para la implementación de políticas locales de salud para inmigrantes en la zona norte de la región metropolitana" (DEMOSCOPICA, 2009), la población inmigrante regular accede con igualdad de derechos y servicios que la población nacional a la Salud Pública. En cambio, los/as inmigrantes en condición de irregularidad -con excepción de los/as menores de 18 años, mujeres embarazadas, y casos de urgencia- están 
imposibilitados de acceder a prestaciones en el sistema de salud.

En el año 2008, a partir de la creación de un grupo de trabajo especializado para la toma decisiones en materia de salud migrante, surgieron una serie de iniciativas con el objetivo de mejorar el acceso a los servicios de salud, especialmente en lo grupos en condición de ilegalidad. Actualmente, se cuenta con la circular "Atención en salud de población inmigrante en riesgo social y situación de permanencia no regular", y las garantías devenidas del Ordinario $\mathrm{n}^{\circ} 3229$ del Ministerio de Salud, con el que se garantiza el derecho a la salud para la población inmigrante infantil, independiente de la condición jurídica de sus padres o personas a su cargo. De esta forma, la atención se realiza por medio de instancias como el "Programa de Salud del Niño y Mujer Embarazada" el cual habilita el acceso a atención sanitaria a la población inmigrante no regular (DEMOSCOPICA, 2009).

No obstante, si bien han existido avances en la materia desde la creación de dicho grupo de trabajo a nivel ministerial, estudios recientes han revelado discriminación por parte de los agentes de salud hacia los inmigrantes, quienes permanentemente son "etnizados" bajo el modelo de discriminación y exclusión que comúnmente se les atribuye a los residentes de origen peruano (Liberona, 2012).

En salud mental, específicamente, los escasos estudios realizados en Chile han determinado que los extranjeros encuentran una serie de barreras de carácter: a) administrativo/legal -condición de legalidad/ilegalidad; b) falta de conocimiento del sistema de salud y sus procedimientos; y c) de carácter cultural que les impiden acceder una atención de calidad (Alvarado, 2008; Rojas et al., 2011).

\section{Metodología}

La investigación corresponde a un estudio de carácter exploratorio-descriptivo que tiene por objetivo analizar el estado actual de la investigación en Chile en temáticas de salud mental en población inmigrante infanto-juvenil.

La información fue recolectada de seis revistas de Psicología, caracterizadas por su alto nivel de indexación y/o tradición en el mundo académico local. En tal sentido, se revisaron los artículos publicados en las revistas Terapia Psicológica (con indexación ISI, es editada por la Sociedad Chilena de Psicología Clínica desde 1982); revista PSYKHE (con indexación
SCIELO, es editada por la Pontificia Universidad Católica de Chile); revista Psicoperspectivas (con indexación SCIELO, es editada por la Pontificia Universidad Católica de Valparaíso desde el 2002); Revista de Psicología de la Universidad de Chile (con indexación LATINDEX, es editada por dicha casa de estudios desde 1990); Revista Salud \& Sociedad (con indexación LATINDEX es arbitrada por la Universidad Católica del Norte, Universidad de Talca, y la Universidad de Tarapacá); y por último; la Revista de Psicología PRAXIS (sin indexación, editada por la Facultad de Psicología de la Universidad Diego Portales desde 1999).

Posteriormente, se elaboró una base de datos con el total de artículos que aparecen por volumen en cada revista. Además, se descartaron los escritos que eran parte de las revistas pero que no correspondían a la sección de artículos (por ej. reseñas de libros). Del total de artículos se seleccionó a aquellos que hacían alusión explícita a la temática migratoria en las secciones: título, resumen y palabras claves. En dichas secciones se buscaron las palabras: "migración", "migrantes", "inmigración" e "inmigrantes".

Los escritos seleccionados se constituyeron en el universo específico a estudiar. Para cada uno se confeccionó una planilla que clasificó la información según: a) tipo de investigación; b) proyecto asociado; c) apoyo institucional; d) nivel de análisis; e) población objetivo; y f) contexto de estudio.

De acuerdo al "tipo de investigación", los artículos fueron clasificados en: a) teóricos: aquellos que realizan elaboraciones teóricas en torno a los problemas migratorios; y b) empíricos: aquellos que realizan investigaciones empírica de corte cuantitativo y/o cualitativo.

Respecto a la categoría "proyecto asociado", se construyeron dos subcategorías: a) con proyecto asociado: aquellas investigaciones adscritas a un proyecto de investigación (FONDECYT, FONIS, etc.); y sin proyecto asociado: aquellas investigaciones que no son parte o no explicitan su adscripción a un proyecto de investigación.

En relación al "apoyo institucional", los artículos fueron ordenados en base al apoyo que recibieron las investigación (financiamiento u otro) según: a) estatales: apoyo a través de recursos públicos para la realización del estudio; b) privados: apoyo a través de recursos privados pertenecientes a instituciones privadas; c) mixtos: apoyo tanto estatal como privado; d) sin especificar: apoyo no explicitado en el artículo o estudio sin apoyo. 
La categoría "nivel de análisis" fue divida en tres sub-categorías: a) nivel microsocial: estudios orientados a nivel local, es decir, de personas y/o su relación con determinados grupos; b) nivel meso-social: estudios orientados a comunidades u organizaciones migrantes; y c) nivel macro-social: estudios referidos a temáticas globales relativas a la migración (aspectos políticos, económicos, estatales, etc.).

Respecto a la categoría "población objetivo", la información se organizó según el tipo de población estudiada: a) peruana; b) argentina; c) colombiana; d) chilena e) otras nacionalidades; mixto (por ej. estudios con poblaciones de dos o más países); y no especificada.

Por último, la categoría "contexto de estudio" permitió organizar la información según la realidad a la cual se abocó la investigación. En tal sentido las subcategorías fueron: a) local (artículos situados en la realidad chilena con población inmigrante o chilena); b) internacional (artículos que hacen referencia al fenómeno migratorio fuera del contexto nacional); c) mixta (artículos que apuntan al contexto nacional e internacional); y d) no especificada (artículos en los que no se hace referencia explícita al contexto del fenómeno migratorio).

\section{Resultados}

La revisión de artículos en las revistas Terapia Psicológica, Psykhe, Psicoperspectivas, Revista de Psicología de la Universidad de Chile, Revista Salud \& Sociedad, y Revista de Psicología Praxis, en el periodo 2003-2013, arrojó un total de 1.094 escritos. Del total de artículos, se encontraron 26 publicaciones relacionadas a la temática migratoria, lo que representa un $2.37 \%$. A continuación, se especifica cada una de estas publicaciones (ver Tabla 1).

\section{Tabla 1}

Publicaciones referidas a la temática de migración en revistas de Psicología en Chile durante el periodo 2003-2013.

\section{Referencia bibliográfica}

1 Luque, C. (2005). Transnacionalismo y enclave territorial étnico en la configuración de la ciudadanía de los inmigrantes peruanos en Santiago de Chile. Praxis, 7(7), 9-26

2 Martínez, J. (2005). Migración internacional: ¿Se puede hacer algo que favorezca a los países de América Latina y el caribe? Praxis, Año 7(7) 27-44

3 Mejido, M. (2005). Postmodernidad, teoría migratoria, y la crítica de la economía política. Praxis, 7(7) 45-50

4 Núñez, L. (2005). Migrantes. ¿Una exportación no tradicional? Praxis, 7(7) 51-58

5 Núñez, L. (2005). Consideraciones en torno a la salud mental en la población migrante peruana en Chile. Praxis, 7(7), 59-66

6 Radovcic, J. (2005). Discriminación étnico-racial en el Chile hacia el bicentenario. Praxis, 7(7), 67-72

7 Ravanal, M. (2005). Los grandes temas de la migración... y los de la casa (los nuestros). Praxis, 7(7), 73-80

8 Schramkowski, B. (2005). Perspectivas sobre integración en Chile. Praxis, 7(7), 81-98

9 Stefoni, C. (2005). Seguridad humana e inmigración en América Latina. Praxis, 7(7), 99-114

10 Godoy, L. (2007). Fenómenos migratorios y género: Identidades femeninas "remodeladas". Psykhe, 16(1), 41-51

11 Navas, L. \& Sánchez, A. (2010). Actitudes de los estudiantes de pedagogía de las regiones del Bio-Bío y la Araucanía de Chile hacia la presencia de niños inmigrantes en la escuela. Análisis diferenciales. Psykhe, 19(1), 47-60.

12 Moctezuma, A.; Torres, D.; Maya, E. (2010). Consideraciones temáticas en torno al estudio contemporáneo de la migración ilegal internacional. Salud \& Sociedad, 1(2), 145-156.

13 Guàrdia, J.; Turbany, J.; Freixa, M.; Honrubia, M.; Carreras, V.; Barrios, M.; Cosculluela, A.; Rifá, J. (2010). Expectativas de permanencia de los inmigrantes de la provincia de Barcelona. Salud \& Sociedad, 1(1), 19-27.

14 Yañez, S.; Cárdenas, M. (2010). Estrategias de aculturación, indicadores de salud mental y bienestar psicológico en un grupo de inmigrantes sudamericanos en Chile. Salud \& Sociedad, 1(1), 51-70.

15 Cárdenas, M.; Gómez, F.; Méndez, L.; Yáñez, S. (2011). Reporte de los niveles de prejuicio sutil y manifiesto hacia los inmigrantes bolivianos y análisis de su relación con variables psicosociales. Psicoperspectivas, 10(1), 125-143

16 Cuestas, F. (2011). Sobre locura y emigración en el mundo globalizado. Psicoperspectivas, 10(1), 21-45.

17 Eguren, J. (2011). La construcción de las comunidades latinoamericanas transnacionales en España. Psicoperspectivas, $10(1), 69-98$. 
18 Espinosa, A.; Herschkowicz, S.; Genna, K. (2011). Correlatos psicológicos de las intenciones y comportamientos migratorios de jóvenes peruanos de clase media y alta. Psicoperspectivas, 10(1), 99-124.

19 Martínez, L.; Peñaranda-Cólera, M.; Vítores, A.; Íñiguez-Rueda, L. (2011). Los locutorios como espacios de integración: las tecnologías de la información y la comunicación en la construcción de redes e identidades. Psicoperspectivas, 10(1), 243-270.

20 Mora, E.; León, F. J. (2011). La globalización del cuidado y sus cadenas: Un estudio de caso. Psicoperspectivas, 10(2), 109-133.

21 Vega, A.; Brindis, L. (2011). La reconfiguración de la imagen pública del migrante a través de las organizaciones migrantes y las remesas colectivas. Psicoperspectivas, 10(1), 46-68.

22 Yuing, T. (2011). Migraciones y administración de la vida en el mundo global. Psicoperspectivas, 10(1), 6-20.

23 Pàmies, J. (2011). Las identidades escolares y sociales de los jóvenes marroquíes en Cataluña (España). Psicoperspectivas, 10(2), 144-168.

24 Méndez, L. y Cárdenas, M. (2012). Hacia la construcción de un modelo comprensivo de análisis de la "situación de inmigración" de mujeres sudamericanas en Chile. Psicoperspectivas, 11(1), 252-272.

25 Basabe, N.; Bobowik, M. (2013). Estatus grupal, discriminación y adaptación en inmigrantes latinoamericanos y africanos en España. Psicoperspectivas, 12(1), 5-29.

26 Bahamondes, P. (2013). Actitudes de los chilenos hacia las orientaciones aculturativas de los inmigrantes peruanos y su relación con variables intergrupales. Praxis. 15(24), 29-55

Tabla 2

Número y porcentaje de artículos publicados en el área de migración (M.), salud mental (S.M.), y población infanto-juvenil (P.I.J.)

Revista $\mathrm{N}^{\circ}$ artículos en $\mathrm{M} . \quad \mathrm{N}^{\circ}$ artículos en M. y S.M. $\mathrm{N}^{\circ}$ artículos en M. y $\mathrm{N}^{\circ}$ artículos en M, S.M., P.I.J. y P.I.J.

\begin{tabular}{lllll} 
Ter. Psicológica & 0 & 0 & 0 & 0 \\
PSYKHE & 2 & 0 & 0 & 0 \\
Psicoperspectivas & 11 & 1 & 2 & 0 \\
Rev. Psicología U. de & 0 & 0 & 0 & 0 \\
Chile & & 1 & 0 & 0 \\
Salud \& Sociedad & 3 & 0 & 1 & 0 \\
Praxis & 10 & 2 & 3 & 0 \\
\hline Total & 26 & &
\end{tabular}

De los 26 artículos referidos a migración, 11 fueron publicados en revista Psicoperspectivas; 10 en la Revista de Psicología PRAXIS; 3 en la revista Salud $\&$ Sociedad; y 2 en la revista PSYKHE. Por su parte, en la Revista Terapia Psicológica y en la Revista de Psicología de la Universidad de Chile no se encontraron artículos relativos a la temática. Por otro lado, se identificaron 2 artículos dedicados a la temática migratoria y la salud mental. Sin embargo, no se hallaron escritos relativos a migración, salud mental y población infantojuvenil específicamente (ver Tabla 2).

En relación la categoría "tipo de estudio", se puede observar que en los artículos seleccionados, existe un predominio de publicaciones que dan cuentan de estudios empíricos (53.8\%) por sobre los de tipo teórico (46.1\%) (ver Tabla 3). 
REVISTAS NACIONALES DE PSICOLOGÍA DURANTE EL PERIODO 2003-20I3

Tabla 3.

Número y porcentaje de artículos según tipo de estudio.

\begin{tabular}{lll}
\hline Revista & Teórico & Empírico \\
\hline & & \\
Terapia Psicológica & 0 & 0 \\
Psykhe & 1 & 1 \\
Psicoperspectivas & 3 & 8 \\
Rev. Psicología U. de Chile & 0 & 0 \\
Salud \& Sociedad & 1 & 2 \\
Praxis & 7 & 3 \\
TOTAL & 12 & 14 \\
Porcentaje & $46.1 \%$ & $53.8 \%$ \\
\hline
\end{tabular}

Tabla 4.

Número y porcentaje de artículos según adscripción a proyecto

\begin{tabular}{lll}
\hline Revista & Con proyecto asociado & Sin proyecto asociado \\
\hline Terapia Psicológica & 0 & 0 \\
Psykhe & 0 & 2 \\
Psicoperspectivas & 3 & 8 \\
Rev. Psicología U. de Chile & 0 & 0 \\
Salud \& Sociedad & 0 & 3 \\
Praxis & 2 & 8 \\
TOTAL & 5 & 21 \\
Porcentaje & $19.2 \%$ & $80.7 \%$ \\
\hline
\end{tabular}

Respecto a la adscripción de dichas publicaciones a proyectos de investigación (por ej. FONDECYT, FONIS, u otros), se constata que sólo 5 de los 26 artículos seleccionados se encuentran asociados a algún proyecto de investigación. Por el contrario, 21 publicaciones (80.7\%) no están adscritas a ningún proyecto (ver Tabla 4).

En relación a la categoría "apoyo institucional", se evidencia que la mayor parte de los artículos no recibieron apoyo institucional (65.3\%). Sin embargo, se confirma la presencia de 9 publicaciones con apoyo institucional, las cuales se subdividen en 7 con apoyo estatal, una con apoyo privado y una con apoyo de carácter mixto (ver Tabla 5).

Tabla 5.

Número y porcentaje de artículos según tipo apoyo institucional

\begin{tabular}{lllll}
\hline & & & & Sin especificación \\
\hline Revista & Estatal & Privado & Mixto & 0 \\
Perapia Psicológica & 0 & 0 & 0 & 2 \\
Psicoperspectivas & 0 & 0 & 0 & 7 \\
Rev. Psicología U. de Chile & 0 & 0 & 0 & 0 \\
Salud \& Sociedad & 2 & 0 & 0 & 1 \\
Praxis & 1 & 0 & 0 & 7 \\
TOTAL & 7 & 1 & 1 & 17 \\
Porcentaje & $26.9 \%$ & 1 & 1 & $65.3 \%$ \\
\hline
\end{tabular}

Respecto a la categoría "nivel de análisis", se constata que los estudios que apuntan a nivel micro-social representan un $30.7 \%$ del total de las investigaciones relativas a la temática migratoria. Por otro lado, los estudios orientados a nivel meso y macro-social comparten igual proporción con un 34.6\% (ver Tabla 6). 
Tabla 6.

Número y porcentaje de artículos según el nivel de análisis de la investigación

\begin{tabular}{llll}
\hline Revista & Microsocial & Mesosocial & Macrosocial \\
\hline Terapia Psicológica & 0 & 0 & 0 \\
Psykhe & 2 & 0 & 0 \\
Psicoperspectivas & 3 & 7 & 1 \\
Rev. Psicología U. de Chile & 0 & 0 & 0 \\
Salud \& Sociedad & 2 & 0 & 1 \\
Rev. Psicología Praxis & 1 & 2 & 7 \\
TOTAL & 8 & 9 & 9 \\
Porcentaje & $30.7 \%$ & $34.6 \%$ & $34.6 \%$ \\
\hline
\end{tabular}

Tabla 7.

Número y porcentaje de artículos según población objetivo

\begin{tabular}{llllllll}
\hline Revista & Peruana & Argentina & Colombiana & Chilena & $\begin{array}{l}\text { Otras } \\
\text { nacionalidades }\end{array}$ & $\begin{array}{l}\text { Mixto } \\
\text { No } \\
\text { especificada }\end{array}$ \\
\hline Terapia Psicológica & 0 & 0 & 0 & 0 & 0 & 0 & 0 \\
Psykhe & 0 & 0 & 0 & 1 & 0 & 1 & 0 \\
Psicoperspectivas & 1 & 0 & 0 & 1 & 1 & 5 & 3 \\
Rev. Psicología U. de Chile & 0 & 0 & 0 & 0 & 0 & 0 & 0 \\
Salud \& Sociedad & 0 & 0 & 0 & 0 & 0 & 2 & 1 \\
Rev. Psicología Praxis & 2 & 0 & 0 & 1 & 0 & 1 & 6 \\
TOTAL & 3 & 0 & 0 & 3 & 1 & 9 & 10 \\
Porcentaje & $11.5 \%$ & $0 \%$ & $0 \%$ & $11.5 \%$ & $3.8 \%$ & $34.6 \%$ & $38.4 \%$ \\
\hline
\end{tabular}

Tabla 8.

Número y porcentaje de artículos según contexto de estudio

\begin{tabular}{lllll}
\hline Revista & Local & Internacional & Mixto & No especificada \\
\hline Terapia Psicológica & 0 & 0 & 0 & 0 \\
Psykhe & 1 & 0 & 1 & 0 \\
Psicoperspectivas & 2 & 7 & 0 & 2 \\
Rev. Psicología U. de Chile & 0 & 0 & 0 & 0 \\
Salud \& Sociedad & 1 & 1 & 0 & 1 \\
Rev. Psicología Praxis & 6 & 2 & 0 & 2 \\
TOTAL & 10 & 10 & 1 & 5 \\
Porcentaje & $38.4 \%$ & $38.4 \%$ & $3.8 \%$ & $19.2 \%$ \\
\hline
\end{tabular}


En relación a la categoría "población objetivo", se constata que la mayor parte de las publicaciones no cuenta con una población objetivo definida. Tales investigaciones representan el $38.4 \%$. Las publicaciones restantes toman como población objetivo a peruanos/as (11.5\%), chilenos/as (11.5\%) y población mixta $(34.6 \%)$. Es importante destacar la inexistencia de publicaciones relativas a población argentina y/o colombiana (ver Tabla 7).

Por último, de los 26 artículos seleccionados, se evidencia que sólo un $38.4 \%$ corresponde a investigaciones abocadas al ámbitos local. Por otro lado, un 38.4\% apunta a un contexto internacional; un $3.8 \%$ contempla tanto aspectos nacionales como internacionales; y un $19.2 \%$ de las investigaciones no explicita el contexto donde se sitúa el análisis (ver Tabla 8).

\section{Conclusiones}

Los resultados revelan el escaso número de publicaciones referidas al tema migratorio en revistas de Psicología a nivel nacional. De un total de 1.094 artículos contabilizados en la búsqueda, sólo 26 escritos abordan aspectos relativos a lo migratorio. De estos, dos tratan la temática de inmigración y salud mental; tres apuntan a lo migratorio y la población infanto-juvenil; mientas que ninguno hace referencia a la salud mental de la población infanto-juvenil inmigrante.

Los artículos sobre migración publicados en las revistas de Psicología revisadas tienden a ser: de carácter empíricos por sobre teóricos; sin proyectos de investigación asociados; con escaso apoyo estatal; sin estar dirigidos a una población específica (o enfocados solamente en población peruana y/o chilena); y sólo en una menor proporción contextualizados a la realidad chilena. Asimismo, tal como señalan estudios previos (Jensen, 2008), las investigaciones tienden a estar enfocadas en problemáticas macro y meso-sociales $(69.2 \%)$ en desmedro de los estudios a nivel microsocial $(30.7 \%)$.

De este modo, se confirma el hecho de que temáticas como la salud mental en población infanto-juvenil inmigrante, tienden a ser invisibilizadas o no consideradas en los estudios que regularmente se llevan a cabo en Psicología en torno al tema migratorio.

Si bien las mediciones censales de los años 1992 y 2002 ya mostraban un crecimiento sostenido del flujo migratorio, la producción de conocimiento en la temática y su posterior materialización en artículos en revistas especializadas en Psicología a nivel nacional, ha estado lejos de dar respuesta a los nuevos problemas que demanda la realidad local. En efecto, las escasas publicaciones en la materia, particularmente en población infanto-juvenil, muestran las casi nulas referencias con las cuales la disciplina puede contar para el trabajo en estas temáticas.

En tal sentido, los aportes disponibles en torno a lo migratorio, la vida cotidiana, y los aspectos subjetivos asociados, han emanado de otras disciplinas pertenecientes a las Ciencias Sociales, como es el caso de la Sociología, principalmente. Los estudios de Tijoux (2013; 2013b; 2012); Stefoni (2003, 2008 et al., Stefoni \& Fernández, 2012), entre otros, son algunos de ellos. Por otro lado, los recientes estudios de Alvarado (2008) y Rojas et al. (2011), desde el campo de la Psiquiatría y la Salud Pública, han informado sobre el estado actual de la salud mental en ciertos grupos inmigrantes.

Investigar y publicar en la materia se vuelve prioritario si se considera, por un lado, la evidencia que respalda que las segundas generaciones de inmigrantes tienden a igualar las tasas de prevalencia de cuadros psicopatológicos de los países de acogida (Breslau et al., 2007); y por otro, las elevadas tasas que se presentan en Chile de trastornos mentales en población infantojuvenil (Vicente, Saldavia, de la Barra, Kohn, Pihan, Valdivia, Rioseco, Melipillan, 2012).

Asimismo, son necesarias aproximaciones de carácter transdisciplinar que permitan pensar tanto la subjetividad de niños/as y adolescentes inmigrantes como los determinantes sociales de la salud mental involucrados (Ingleby, 2012). Dichas aproximaciones brindarían la posibilidad de indagar en la salud mental teniendo en consideración los aspectos subjetivos que se despliegan en las mutuas relaciones que establecen población inmigrante e institución (por ej. escuela, servicios de salud, Estado, etc.).

\section{Referencias}

Acosta, E. (2014). Valorar los cuidados al estudiar las migraciones: La crisis del trabajo de cuidado y la feminización de la inmigración en Chile. En C. Stefoni (2012). Mujeres Inmigrantes en Chile. ¿Mano de obra o trabajadoras con derechos? Santiago: Ediciones Alberto Hurtado.

Arriagada, I. \& Moreno, M. (2012). La constitución de cadenas globales de cuidado y las condiciones laborales de las trabajadoras peruanas en Chile. En C. Stefoni (Ed.), Mujeres inmigrantes en Chile. ¿Mano de obra o trabajadoras con derechos? (pp. 149-192). Santiago: Ediciones Alberto Hurtado.

Alvarado, R. (2008). Salud mental en inmigrantes. Revista Chilena de Salud Pública, 12(1), 37-41. doi:10.5354/0719-5281.2008.2075 
Breslau, J., Aguilar-Gaxiola, S., Borges, G., Kendler, K., Su, M. \& Kessler, R. (2007). Risk for psychiatric disorder among immigrants and their US-born descendants: evidence from the National Comorbidity Survey Replication. The Journal of Nervous and Mental Disease, 195(3), 189-95. doi:10.1097/01. nmd.0000243779.35541.c6

Cano, V. \& Soffia, M. (2009). Los estudios sobre migración internacional en Chile: apuntes y comentario para una agenda de investigación actualizada. Papeles de Población, 15(61), 129167. Recuperado de http://www.scielo.org.mx/pdf/pp/v15n61/ v15n61a7.pdf

Castles, S. \& Miller, M. (2004). La era de la migración. Movimientos internacionales de población en el mundo moderno. México: Universidad Autónoma de Zacatecas.

Cortez, A. (2004). Integración de los niños y niñas inmigrantes al sistema escolar chileno: Obstáculos y desafíos. En Colectivo Sin Fronteras (Ed.), Niños y niñas inmigrantes en Chile: derechos y realidades. Santiago: CSF-Fundación Anide.

DEMOSCOPICA (2009). Informe final: Diagnóstico y factibilidad global para la implementación de políticas locales de salud para inmigrantes en la zona norte de la región metropolitana. Santiago, Chile.

Harker, K. (2001). Immigrant generation, assimilation and adolescent psychological well-being. Social Force, 79(3), 969-1004. doi:10.1353/sof.2001.0010

Hevia, P. (2009). Niños inmigrantes peruanos en la escuela chilena. (Memoria Antropología Social). Universidad de Chile, Santiago.

Ingleby, D. (2012). Ethnicity, Migration and the 'social determinants of health' Agenda. Psychosocial interventionm, 21(3), 331-341. doi:10.5093/in2012a29

Instituto Nacional de Estadísticas. (2013). Censo 2012. Instituto Nacional de Estadísticas, Gobierno de Chile, Chile.

Instituto Nacional de Estadísticas. (2003). Censo 2002. Instituto Nacional de Estadísticas, Gobierno de Chile, Chile.

Jensen, M. (2008). Inmigrantes en Chile: La exclusión vista desde la política migratoria chilena. En Asociación Latinoamericana de Población (Eds.), Temáticas migratorias actuales en América Latina: remesas, políticas y emigración (pp. 105-130). Brasil: Trilce.

Liberona, N. (2012). De la alterisación a la discriminación en un sistema público de salud en crisis: conflictos interétnicos a propósito de la inmigración sudamericana en Chile. Revista de Ciencias Sociales, 28, 19-38. Recuperado de http://www.revistacienciasociales.cl/archivos/revista28/pdf/rcs28_art01.pdf

MIDEPLAN (2011). CASEN 2011: Resultados Nacionales. Ministerio de Planificación y Cooperación, Gobierno de Chile, Chile.

MIDEPLAN (2006). CASEN 2006: Resultados Nacionales. Ministerio de Planificación y Cooperación, Gobierno de Chile, Chile.

Ministerio del Interior. Departamento de Extranjería y Migración. (2009). Informe de Permisos Otorgados y Estimación de población de extranjeros en Chile a diciembre de 2009. DEM-Ministerio del Interior. Gobierno de Chile. Chile.

Pavez, I. (2013). Los significados de "ser niña y niño migrante": conceptualizaciones desde la infancia peruana en Chile. Polis. Revista Latinoamericana, 35, 1-19. Recuperado de http://polis. revues.org/9304

Pavez, I. (2010). Los derechos de las niñas y los niños peruanos en Chile. La infancia como un nuevo actor migratorio. Revista Enfoques Ciencia Política y Administración Pública, 8(12) 2751. Recuperado de http://www.politicaygobierno.cl/documentos/ enfoques/12/art_Iskra_Pavez.pdf
Pérez, A. (2010). Cadenas Globales de Cuidado. ¿Qué derechos para un régimen global de cuidados justos? Instituto Internacional de Investigaciones y Capacitación de las Naciones Unidas para la Promoción de la Mujer. UN-INSTRAW: República Dominicana.

Pérez, A. (2009). Miradas globales a la organización social de los cuidados en tiempos de crisis I: ¿Qué está ocurriendo? Serie Género, Migración y Desarrollo. Documentos de trabajo $\mathrm{N}^{\circ} 5$. INSTRAW.

Reyes, C. (2007). Niños y niñas sin Fronteras en las escuelas. En Colectivo Sin Fronteras. Niños y niñas migrantes. Políticas públicas, integración e interculturalidad. Santiago: Editorial Colectivo Sin Fronteras-Fundación Anide-KinderNotHilfe.

Rojas, G., Fritsch R., Castro, A., Guajardo, V., Torres, P. \& Díaz, B. (2011). Trastornos mentales comunes y uso de servicios de salud en población inmigrante. Revista Médica de Chile, 139(10), 12981304. doi: 10.4067/S0034-98872011001000008

Stefoni, C. \& Fernández, R. (2012). Mujeres inmigrantes en el trabajo doméstico: Entre el servilismo y los derechos. En Mujeres Inmigrantes en Chile. ¿Mano de obra o trabajadoras con derechos? Santiago: Ediciones Alberto Hurtado.

Stefoni, C.; Acosta, E.; Gaymer, M.; Casas-Cordero, F. (2008). Niños y niñas inmigrantes en Santiago de Chile. Entre la integración y la exclusión. Santiago: OIM-Universidad Alberto Hurtado.

Stefoni, C. (2003). Inmigración y ciudadanía: La formación de comunidades peruanas en Santiago y la emergencia de nuevos ciudadanos. Política, 43, 319-336. Recuperado de http://www. redalyc.org/pdf/645/64504313.pdf

Tijoux, M. E. (2013). Niños(as) marcados por la inmigración peruana: estigma, sufrimientos, resistencias. Convergencia Revista de Ciencias Sociales, 20(61), 83-104. Recuperado de http://www. scielo.org.mx/pdf/conver/v20n61/v20n61a4.pdf

Tijoux, M.E. (2013b). Las escuelas de la inmigración en la ciudad de Santiago. Elementos para una educación contra el racismo. Polis. Revista Latinoamericana, 12(35), 287-307. doi:10.4067/ S0718-65682013000200013

Tijoux, M. E. (2012). Negando al "otro": El constante sufrimiento de los inmigrantes peruanos en Chile. En C. Stefoni (Ed.), Mujeres inmigrantes en Chile. ¿Mano de obra o trabajadoras con derechos? (pp. 17-42). Santiago: Ediciones Alberto Hurtado.

UNICEF. (2004). Informe anual de actividades 2004. Santiago: UNICEF.

Vázquez-De Kartzow, R. (2010). El mundo está cambiando. Viejos y nuevos actores de la epidemiología en tiempos de globalización. En L. Agar Corbinos (Coord.), Migraciones, salud y globalización: Entrelazando miradas (pp. 25-36). Santiago: Editorial Biplano. OIM/OPS/MINSAL.

Vásquez, M. E. (2007). Intervención local y comunitaria para la integración social de los inmigrantes. En Colectivo Sin Fronteras (2007). Niños y niñas migrantes. Políticas públicas, integración e interculturalidad. Santiago: Editorial Colectivo Sin FronterasFundación Anide-KinderNotHilfe.

Vicente, B., Saldavia, S., de la Barra, F., Kohn, R., Pihan, R., Valdivia, M., ... \& Melipillan, R. (2012). Prevalence of child and adolescent mental disorders in Chile: a community epidemiological study. Journal of child psychology and psychiatry, 53(10), 1026-1035. doi:10.1111/j.1469-7610.2012.02566.x

Zolkowska, K., Cantor-Graae, E. \& McNeil, T. (2001). Increased rates of psychosis among immigrants to Sweden: is migration a risk factor for psychosis? Psychological Medicine, 31(4), 669-678. Recuperado de http://lup.lub.lu.se/luur/download?func=downloa dFile\&recordOId=131487\&fileOId=624217. 